\title{
Morning surge in blood pressure and sympathetic activity in Mongolians and Han Chinese: a multimodality investigation of hypertension and dyssomnia
}

\author{
Guanhua Huang ${ }^{1}$, Xiaoming Yang ${ }^{2}$, Jing Huang ${ }^{\text {Corresp. } 1}$ \\ ${ }^{1}$ Department of Cardiology, The Second Affiliated Hospital of Chongqing Medical University, Chongqing, Chongqing, China \\ 2 Department of Cardiology, The Second Affiliated Hospital of Baotou Medical College, Baotou, Inner Mongolia, China \\ Corresponding Author: Jing Huang \\ Email address: huangjing_003@sina.com
}

ABSTRACT Background. Hypertension and dyssomnia are increasing significantly in Mongolians, and the related factors of ethnic differences in hypertension and dyssomnia between Mongolians and Han Chinese are unclear. This study examined the relationship of morning surge in blood pressure (MBP) with ethnicity, sleep situation, and sympathetic activity throughout the day. Methods. Of 692 hypertensive patients screened, 202 subjects with dyssomnia were selected. They were then divided into Mongolian $(n=87)$ and Han $(n=115)$ groups. The differences in dyssomnia, 24-hour blood pressure, and urinary catecholamine were analyzed in all subjects; they were then further divided according to the degree of dyssomnia (low, moderate, and severe) to determine the differences in blood pressure and catecholamine. Results. Mongolians had lower history of smoking, daytime dysfunction, nocturnal heart rates, and dopamine levels, but their body mass index, triglyceride, fasting glucose, morning surge in systolic blood pressure (MSBP), nocturnal systolic blood pressure (NSBP), nocturnal diastolic blood pressure, daytime systolic blood pressure, daytime heart rates, and dopamine level (D-DA) were higher than those of Han Chinese. With aggravation of dyssomnia, MSBP, NSBP, D-NE, daytime epinephrine, and D-DA of Mongolians and Han Chinese increased gradually, but the rate of increase was faster in the latter $(p<0.05)$. D-DA was entered into the MSBP regression model of Mongolians (intercept, $157 \mathrm{mmHg}$ ), whereas D-DA and D-NE were entered into the MSBP regression model of Han Chinese (intercept, $142 \mathrm{mmHg}$ ). Conclusion. Worsened dyssomnia induces higher MSBP and augments sympathetic excitability in Mongolians and Han Chinese. Mongolians with hypertension and dyssomnia had higher MSBP baseline and D-DA but lower N-DA. With increase in D-DA, MSBP in Han and Mongolian patients increased gradually. 
1 Morning surge in blood pressure and sympathetic activity in Mongolians and

2 Han Chinese: a multimodality investigation of hypertension and dyssomnia

3

5 Chongqing, China

6 bepartment of Cardiology, The Second Affiliated Hospital of Baotou Medical College, Baotou,

7 Inner Mongolia, China

8 *Corresponding author: Jing Huang, PhD, Department of Cardiology, The Second Affiliated

9 Hospital of Chongqing Medical University, No. 74 Linjiang Road, Chongqing 400010, China.

10 Phone: +86-13508312022, E-mail: huangjingcqmu@126.com

11 Subjects Hypertension and Dyssomnia

12 Number of words: 3073 
14

15

\section{ABSTRACT}

Background. Hypertension and dyssomnia are increasing significantly in Mongolians, and the related factors of ethnic differences in hypertension and dyssomnia between Mongolians and Han Chinese are unclear. This study examined the relationship of morning surge in blood pressure (MBP) with ethnicity, sleep situation, and sympathetic activity throughout the day. Methods. Of 692 hypertensive patients screened, 202 subjects with dyssomnia were selected. They were then divided into Mongolian $(n=87)$ and Han $(n=115)$ groups. The differences in dyssomnia, 24-hour blood pressure, and urinary catecholamine were analyzed in all subjects; they were then further divided according to the degree of dyssomnia (low, moderate, and severe) to determine the differences in blood pressure and catecholamine.

Results. Mongolians had lower history of smoking, daytime dysfunction, nocturnal heart rates, and dopamine levels, but their body mass index, triglyceride, fasting glucose, morning surge in systolic blood pressure (MSBP), nocturnal systolic blood pressure (NSBP), nocturnal diastolic blood pressure, daytime systolic blood pressure, daytime heart rates, and dopamine level (D-DA) were higher than those of Han Chinese. With aggravation of dyssomnia, MSBP, NSBP, D-NE, daytime epinephrine, and D-DA of Mongolians and Han Chinese increased gradually, but the rate of increase was faster in the latter $(\mathrm{p}<0.05)$. D-DA was entered into the MSBP regression model of Mongolians (intercept, $157 \mathrm{mmHg}$ ), whereas D-DA and D-NE were entered into the MSBP regression model of Han Chinese (intercept, $142 \mathrm{mmHg}$ ).

Conclusion. Worsened dyssomnia induces higher MSBP and augments sympathetic excitability in Mongolians and Han Chinese. Mongolians with hypertension and dyssomnia had higher MSBP baseline and D-DA but lower N-DA. With increase in D-DA, MSBP in Han and Mongolian patients increased gradually. 


\section{INTRODUCTION}

Evidence from previous studies suggests that dyssomnia can increase the incidence of high blood pressure, even if it is a single factor that can lead to high blood pressure in a given environment for quite a time (Joyner et al. 2010). However, discomfort caused by elevated blood pressure at night can also lead to sleep disorder (Walters \& Rye 2009; Wing et al. 2010; Zou et al. 2009). Hypertension and dyssomnia seem to be reciprocal causations under certain conditions. At present, patients with these conditions are increasing. However, despite the evidence, physicians often ignore sleep disorders and their possible relationship with hypertension.

Elevated morning surge in blood pressure (MBP) is undoubtedly a major sign of these two diseases, which could increase damage to organs affected by cardiovascular and cerebrovascular diseases and increase morbidity and mortality (Hoshide et al. 2016; Lyhne et al. 2015; Sheppard et al. 2015). Meanwhile, some studies have shown that elevated MBP is related to sympathetic overactivity and the destruction of the body's normal blood pressure rhythm, and it is associated with alterations in sympathetic excitability during sleep (Johnson et al. 2016; Okada et al. 2013).

In China, different ethnic groups have different levels of blood pressure, and the prevalence of hypertension is also different. Mongolians are the ninth largest minority in China, and their incidence of hypertension is higher than that of Han Chinese (Okada et al. 2013). At present, hypertension and dyssomnia in Mongolians are increasing significantly (Li et al. 2016a), and the related factors of ethnic differences in hypertension and dyssomnia between these two populations are unclear.

Thus, we conducted a prospective cross-sectional study to confirm the hypothesis that Mongolian patients with hypertension and dyssomnia have more obvious increase in sympathetic excitability, higher MBP, and closer correlation between sympathetic activity and MBP, compared with Han patients.

\section{MATERIAL AND METHODS}

\section{Study participants}

Participants were recruited from the Second Affiliated Hospital of Baotou Medical College in the 
65

66

67

68

69

70

71

72

city of Baotou, Inner Mongolia, by posting study flyers in cardiovascular clinics and wards. Study samples who met eligibility criteria were screened from a total of 692 participants (Figure 1). The program was approved by the Ethics Committee of the hospital (Ethical Application Ref: 012-2016), and all subjects provided written informed consent.

Hypertensive Mongolian and Han patients $\geq 18$ years or older with systolic blood pressure $(\mathrm{SBP}) \geq 140 \mathrm{mmHg}$ and/or diastolic blood pressure $(\mathrm{DBP}) \geq 90 \mathrm{mmHg}$ and blood pressure $<180 / 110 \mathrm{mmHg}$ measured three times or more on different days and have not taken any medicines during the week were included.

Patients with secondary hypertension, stroke, coronary heart disease, diabetes mellitus, severe arrhythmia, heart failure, valvular disease, liver and kidney dysfunction, mental disorders, tumor, respiratory diseases, hyperthyroidism, connective tissue disease, or infection; those who had surgery in the past 3 months; those who were pregnant or breastfeeding; those who cannot independently complete the questionnaire independently; and those who were unwilling to participate in the clinical study were excluded.

To avoid interference factors as much as possible, patients taking any medicines within a week, including antihypertensive drugs and drugs for the treatment of dyssomnia, were excluded. Patients with $\mathrm{SBP} \geq 180 \mathrm{mmHg}$ or $\mathrm{DBP} \geq 110 \mathrm{mmHg}$ were also excluded to ensure their safety during examination.

\section{Study methods}

All subjects answered the Pittsburgh sleep quality index (PSQI) questionnaire, with PSQI $\geq 7$ points being enrolled in this study. Selected subjects supplied their demographic characteristics, and underwent blood biochemistry analysis, 24-hour urinary catecholamine detection, and 24hour ambulatory blood pressure monitoring (ABPM). Blood biochemical analysis, 24-hour urine catecholamine detection, and ABPM were performed on the same day. Fasting blood was drawn from the subjects before 07:00. The subjects proceeded to the laboratory to get containers and preservatives for collection of 24-hour urine catecholamines and then underwent ABPM. The next day, at the appointed time, subjects returned for another ABPM and submission of urine 
92 specimens.

93

94

\section{PSQI questionnaire}

All subjects were investigated by PSQI, which is a standardized subjective questionnaire used to retrospectively rate sleep quality during the past 4 weeks (Buysse et al. 1989). This instrument is composed of seven components: sleep time, sleep quality, sleep efficiency, sleep latency, sodium amytal use, sleep disturbance and daytime dysfunction. Each category can score up to 3 points, and the instrument's total score can range from 0 to 21 points: $0-6$, no sleep disorder; $7-11$, mild sleep disorder (PSQI-I); 12-16, moderate sleep disorder (PSQI-II); 17-21, severe sleep disorder (PSQI-III). PSQI $\geq 7$ is the diagnostic standard of dyssomnia (Buysse et al. 1989).

\section{Demographic feature collection}

The demographic characteristics of all selected subjects were collected (name, age, sex, ethnicity, residence, nature of work, exercise, height, weight, and history of smoking). The body mass index (BMI) was computed as weight $/$ height $^{2}\left(\mathrm{~kg} / \mathrm{m}^{2}\right)$.

\section{Blood index detection}

Morning fasting blood was used for the biochemical examination of fasting glucose (FG), triglyceride (TG), total cholesterol (CHO), low-density lipoprotein cholesterol (LDL-C), highdensity lipoprotein cholesterol (HDL-C), aspartate alanine transaminase (AST), alanine transaminase (ALT), and creatinine $(\mathrm{Cr})$.

\section{ABPM}

A noninvasive blood pressure monitor (HEM-9000AI; Omron, Kyoto, Japan) that can record the corresponding blood pressure and heart rate was used for the ABPM of subjects every 30 minutes during daytime (06:00-22:00) and every 60 minutes during nighttime (22:00-06:00). Daytime systolic and diastolic, nocturnal systolic and diastolic, morning surge in systolic and diastolic blood pressure (DSBP, DDBP, NSBP, NDBP, MSBP, and MDBP, respectively) and the daytime and nocturnal mean heart rates (D-HR and N-HR, respectively) were recorded. Effective blood pressure and heart rate data should be greater than $85 \%$ of the set number; otherwise, they were retested. 
119

120

121

122

123

124

125

126

127

128

129

130

131

132

133

134

135

136

137

138

139

140

141

142

143

144

145

\section{Measures of 24-hour urine catecholamine}

Subjects collected 24-hour urine samples during two periods (day and night) of the ABPM session. Each urine sample container had a preservative to prevent deterioration of urine and was stored in portable coolers. Urinary norepinephrine, epinephrine, and dopamine were tested by high-pressure liquid chromatography with electrochemical detection.

\section{Statistical analysis}

Data were expressed as mean \pm standard deviation $(\mathrm{SD})$ for continuous variables and as number (percentage) for categorical variables. To assess ethnic differences, Mann-Whitney U-test was used for continuous non-normally distributed variables and $\chi^{2}$ test was used for categorical variables. Kruskal-Wallis H-test was used to compare blood pressure and catecholamine in the PSQI groups. Spearman's correlations were used to assess the relationships between MSBP and catecholamine. Multiple linear regression analysis was used to determine the regression equation of MSBP and catecholamine in the Mongolian and Han groups. The SPSS 22.0 system (IBM, Armonk, NY, USA) was used for the statistical analysis, and significance was set at $\mathrm{p}<0.05$.

\section{RESULTS}

\section{Patient demographic and clinical characteristics}

A total of 692 hypertensive patients were enrolled in this study, of whom $281(40.61 \%)$ had dyssomnia. Because of incomplete registration of subjects, liver and kidney dysfunctions, nonstandardized ABPM data, and failed urine catecholamine specimens, 69 patients were excluded, and finally, 202 complete data of hypertensive patients with dyssomnia were included in the study (Han, $n=115,56.93 \%$; Mongolians, $n=87,43.07 \%$ ) (Figure 1). The Mongolian group had higher BMI, FG, TG, DSBP, MSBP, NSBP, NDBP, D-HR, and daytime dopamine level (DDA) $(p<0.05)$, but their smoking history, DDBP, N-HR, and N-DA were lower than those of the Han group $(\mathrm{p}<0.05)$ (Table 1). The changes in SBP, DBP, and HR in Mongolian and Han patients during 24 hours were shown in Figure 2.

\section{Demographic and basic characteristics of different PSQI groups}

There was no statistical difference in the three PSQI degrees between the Mongolian and Han 
146

147

148

149

150

151

152

153

154

155

156

157

158

159

160

161

162

163

164

165

166

167

168

169

170

171

172

173

groups in the following aspects: sex, age, residence, nature of work, and movement condition. However, an approximately $50 \%$ incidence rate of hypertension complicated by dyssomnia was found in patients younger than 50 years in these two groups (Table 2).

Sleep time, sleep quality, sleep efficiency, sleep latency, sodium amytal use, sleep disturbance, and PSQI scores had no significant difference between the Mongolian and Han groups, but daytime dysfunction in the Mongolian group was much lower than that of the Han group $(\mathrm{p}<0.01)$ (Table 3).

\section{Comparison of ABPM between the Mongolian and Han patients in different PSQI groups}

In the Han group, with gradual aggravation of dyssomnia, DSBP, NSBP, NDBP, and MSBP also increased gradually, and there were statistically significant differences among the three PSQI groups $(\mathrm{p}<0.01)$. Meanwhile, DDBP and MDBP also increased gradually, but there was no statistical difference (Table 4).

In the Mongolian group, with gradual aggravation of sleep disorder, NSBP and MSBP also increased gradually, and there were statistically significant differences among the three PSQI groups. Although DSBP, DDBP, NDBP, and MDBP also increased gradually, there was no statistical difference (Table 5).

In the PSQI-I group, DSBP, NSBP, NDBP, and MSBP were significantly different between the Mongolian and Han groups. In the PSQI-II group, DSBP, NSBP, and MSBP had statistically significant differences. In the PSQI-III group, blood pressure had no statistical difference (Table 6).

\section{Comparison of 24-hour urinary catecholamine between the Mongolian and Han patients in} different PSQI groups

In the Han group, with gradual aggravation of dyssomnia, D-NE, D-E, D-DA, N-NE, and N-DA were also increasing and had statistically significant differences among the three PSQI groups (Table 4) $(\mathrm{p}<0.01)$.

In the Mongolian group, with gradual aggravation of sleep disorder, N-NE, D-E, and D-DA increasing gradually, but not seen a consistent increasing in D-DA, N-E and N-DA. Meanwhile, with the increase in PSQI degree, the increase in catecholamine was faster in the Han group than 
174 in the Mongolian group (Tables 4 and 5).

175 In the PSQI-I group, D-NE, N-NE, and D-DA were significantly different between the

176 Mongolian and Han groups. In the PSQI-II group, N-DA had statistical difference. In the PSQI-

177 III group, D-NE and N-DA had statistical difference (Table 6).

178 Relationship between MSBP and urinary catecholamine

179 Spearman's correlation of MSBP and D-NE, D-E, D-DA, N-NE, N-E, and N-DA showed that

180 MSBP and D-DA were correlated $(r=0.254, p<0.05$, Figures 3$)$ in the Mongolian group. In the

181 Han group, MSBP was correlated with D-NE, D-E, D-DA, N-NE, and N-DA (r=0.396, r=0.285

$182 \mathrm{r}=0.403, \mathrm{r}=0.309$, and $\mathrm{r}=0.294$, respectively, $\mathrm{p}<0.05$, Figures 3 ). Multivariate linear regression

183 analysis was used to rule out the impact of other confounders (age, sex, BMI, smoking, blood

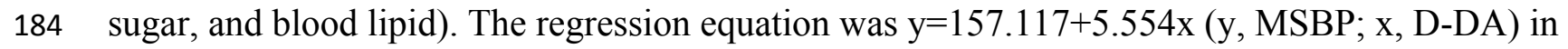

185 the Mongolian group, which indicated that when $1 \mu \mathrm{mol} / \mathrm{L}$ D-DA was added, MSBP increased

186 by an average of $5.554 \mathrm{mmHg}$. In the Han group, the regression equation was

$187 \mathrm{y}=141.730+8.108 \mathrm{x}_{1}+16.984 \mathrm{x}_{2}\left(\mathrm{y}, \mathrm{MSBP} ; \mathrm{x}_{1}, \mathrm{D}-\mathrm{DA} ; \mathrm{x}_{2}, \mathrm{D}-\mathrm{NE}\right)$, which suggested that when 1

$188 \mu \mathrm{mol} / \mathrm{L}$ D-DA was added, MSBP increased by an average of $8.108 \mathrm{mmHg}$, and when $1 \mu \mathrm{mol} / \mathrm{L}^{-1}$

189 D-NE was added, MSBP increased by $16.984 \mathrm{mmHg}$.

190 DISCUSSION

191 Mongolians mainly live in the Inner Mongolia Autonomous Region in China, where Han

192 Chinese and Mongolians account for about $96 \%$ of the total population, and these two ethnic

193 groups have different customs and lifestyle (Okada et al. 2013). There is marked ethnic

194 difference in the prevalence of hypertension. A cross-sectional study in, 2014 showed that the

195 prevalence rate of hypertension in the Inner Mongolia Autonomous Region was higher in

196 Mongolians (31.46\%) than in Han Chinese (27.47\%) (Li et al. 2016a). Previous reports

197 suggested that obesity, dietary history, habit, and genetics were all hypertension-related risk

198 factors in the Mongolian population (Carretero \& Oparil 2000; Vaidya \& Forman 2010).

199 Hypertension is considered as the major cause of disability and death worldwide, especially

200 MSBP, which is associated with coronary heart disease, stroke, and renal dysfunction (Hu et al. 
201

202

203

204

205

206

207

208

209

210

211

212

213

214

215

216

217

218

219

220

221

222

223

224

225

226

227

2012; Kearney et al. 2005). The relationship between BP and the risk of cardio-cerebrovascular disease is continuous - the risk of developing myocardial infarction, heart failure, and stroke increases with higher BP (Li et al. 2008). Mongolians in China were found to have higher prevalence of hypertension and other cardiovascular risk factors than the Han Chinese ( $\mathrm{Li}$ et al. $\underline{2016 \mathrm{~b}}$ ). In addition, the prevalence rates of hypertensive complications such as CVD and stroke are significantly higher in the same geographical area than in the Han Chinese (Zhang et al. $\underline{2007)}$.

\section{Characteristics of Mongolian and Han patients}

As can be seen in our study, Mongolians had higher BMI, FG, and TG levels than Han Chinese, but lower history of smoking, which can probably be attributed to their carnivorous diet and low preference for smoking. In our opinion, elevated BMI, FG, and TG levels depend on dietary history to a great extent and on some habits, such as high-salt diets and alcohol consumption, which are both closely associated with dietary history. Smoking, a commonly accepted hypertension-related factor (Carretero \& Oparil 2000), was lower in the Mongolian group than in the Han group in the present study, which is contrary to the high incidence of hypertension in Mongolians. There were no differences in residence, nature of work, or movement between the two groups, possibly because the recruited addresses were located in a central urban area. In addition, this study showed that even with the same degree of dyssomnia at night, the Mongolian group had lower daytime dysfunction and physical discomfort. We also found that regardless of the level of sleep disorder, the highest incidence of hypertension complicated with dyssomnia was found in patients younger than 50 years in these two groups, which accounted for half of the subjects. Consistent with previous reports, middle-aged, rather than elderly, Chinese with sleep disorder were more likely to be hypertensive than healthy people (Guo et al. 2016). Similar disparities according to age have also been found in other countries (Dean et al. 2012; Lombardi \& Parati 2000).

\section{Sleep and sympathetic nerve activity}

Sleep has been recognized as an essential element of cardiovascular control, because sleep 
228

229

230

231

232

233

234

235

236

237

238

239

240

241

242

243

244

245

246

247

248

249

250

251

252

253

254

disorders are interrelated with increased risk of hypertension, cardiac disease, and stroke

(Cappuccio et al. 2011; Dean et al. 2012; Lombardi \& Parati 2000). Sleep-related hypertension is often masked as a non-dipping status at an early stage. Thereafter, an increase in sympathetic activity results in daytime hypertension and elevated MSBP. Normal nocturnal sleep is characterized by a marked decrease in sympathetic activity and blood pressure, which affects the sympathetic rhythm of blood pressure during the following daytime period (Sayk et al. 2007).

Deeper sleep has been shown to be accompanied by further suppression of the sympathetic function. Lack of an enough deeper sleep period can lead to sympathetic nervous system dysfunction (Yang et al. 2002). Some studies showed that dyssomnia was related to changes in sympathovagal activity, as evidenced by increased catecholamine release and decreased heart rate (Lusardi et al. 1999; Spiegel et al. 1999), which were similar to the findings of our study.

In our study, with the aggravation of sleep disorder, whether in the Mongolian or Han group, DSBP, DDBP, NSBP, NDBP, MSBP, and MDBP gradually increased as well, which was in agreement with previous studies (Haas et al. 2005; Meisinger et al. 2007). Furthermore, the blood pressure of the Han group was more sensitive to the degree of dyssomnia and blood pressure increased more significantly. Meanwhile, with the aggravation of dyssomnia, N-NE, DE, and D-DA increased as well. The catecholamine level of the Han group increased obviously during both daytime and nighttime, whereas the increasing catecholamine of the Mongolian group did not have consistent with the degree of PSQI. Besides, in the PSQI-I group, the catecholamine of the Mongolian group was higher than that of the Han group at both daytime and nighttime; in the PSQI-II group, the catecholamine of the Mongolian group was higher than that of the Han group during daytime, but lower at nighttime; in the PSQI-III group, regardless of time, the catecholamine of the Mongolian group was lower. It seems that the rate of catecholamine secretion was reversed between the two groups with aggravation of sleep disorder. This trend was similar, but not identical, to that of blood pressure in our study. Different degrees of sleep disorder affect the change in MSBP, either in Mongolian or Han patients, and the higher the degree of sleep disorder, the higher the MSBP. Increasing evidence suggests that sleep and 
255

256

257

258

259

260

261

262

263

264

265

266

267

268

269

270

271

272

273

274

275

276

277

278

279

280

281

sleep-related blood pressure control have a significant contribution to the development of hypertension (Carrington et al. 2005; Carrington \& Trinder 2008; Lombardi \& Parati 2000).

\section{Blood pressure and sympathetic nervous activity}

The sympathetic nervous system is thought to play a crucial role in the regulation of blood pressure during daytime and nighttime. During sleep, the baroreceptor reflex is lower, which reduces the sympathetic activity of the heart and blood vessels, eventually leading to a drop in blood pressure (DiBona 2002). However, sympathetic overactivity in sleep disorder causes elevated blood pressure and diurnal blood pressure control dysfunction, which lead to damage of target organs.

Previous research indicated that the sympathetic nervous system had a significant influence on MSBP (Kario et al. 2004);(Hashimoto et al. 2003). Otherwise, individuals with exaggerated MSBP have higher levels of urinary catecholamine excretion (Hashimoto et al. 2003). In our study, there was a positive correlation between D-DA and MSBP in Mongolian and Han patients. Regression analysis showed that D-DA was independently correlated with MSBP. The two sets of the regression intercepts were 157 and $142 \mathrm{mmHg}$ in Mongolians and Han Chinese, respectively, which showed that the change in D-DA affected MSBP and the starting point of MSBP in Mongolian patients was higher by about $15 \mathrm{mmHg}$ than that of Han patients. With increasing D-NE, MSBP was also increasing in Han patients, but this was not seen in Mongolian patients. Studies showed that catecholamine may be pivotal in the racial variation in blood pressure, which suggested that African-Americans had better receptor sensitivity than EuropeanAmericans (Mills et al. 1995; Rutledge 1991). Some observational research have shown that in real life, the level of variations in catecholamine during the day is related to the degree of decrease in blood pressure with sleep (Arita et al. 1996).

\section{Study limitations}

Our study has limitations, such as its small sample size. Additionally, we did not use polysomnography to assess sleep architecture. Furthermore, the Mongolian patients in our study may have changed their habits and customs to a certain extent when they lived in the same area 
282 as the Han Chinese, and high blood pressure therapy may have been underestimated due to

283 inclusion criteria of the study.

\section{CONCLUSIONS}

285 Worsened dyssomnia induces higher MSBP and augments sympathetic excitability in

286

287

288

289

290

291

292

293

294

295

296

297

298

299

300

301

302

303

304

305

306

307

308

309

310

311

312

313

314

315
Mongolians and Han Chinese. However, Mongolian patients have higher baseline of MSBP but lower daytime dysfunction. They also have higher D-DA but lower N-DA. With the increase in D-DA, the MSBP in Mongolian patients also gradually increased, but their rate of increase is lower than that in Han patients. These ethnic characteristics might provide information when deciding on clinical medication. Further studies on the mechanism of underlying genetic factors and pathological routines in the two ethnicities are needed.

\section{ACKNOWLEDGMENT}

We appreciate the staff of the Institute of Hypertension and Biochemical Laboratory in the Second Affiliated Hospital of Baotou Medical College.

\section{REFERENCES}

Arita M, Minami E, Nakamura C, Ueno Y, Nishio I, and Masuyama Y. 1996. Role of the sympathetic nervous system in the nocturnal fall in blood pressure. Hypertension Research 19:195-200.

Buysse DJ, Reynolds CF, 3rd, Monk TH, Berman SR, and Kupfer DJ. 1989. The Pittsburgh Sleep Quality Index: a new instrument for psychiatric practice and research. Psychiatry Research 28:193-213.

Cappuccio FP, Cooper D, D'Elia L, Strazzullo P, and Miller MA. 2011. Sleep duration predicts cardiovascular outcomes: a systematic review and meta-analysis of prospective studies. European Heart Journal 32:14841492. 10.1093/eurheartj/ehr007

Carretero OA, and Oparil S. 2000. Essential hypertension. Part I: definition and etiology. Circulation 101:329-335.

Carrington MJ, Barbieri R, Colrain IM, Crowley KE, Kim Y, and Trinder J. 2005. Changes in cardiovascular function during the sleep onset period in young adults. J Appl Physiol (1985) 98:468-476. 10.1152/japplphysiol.00702.2004

Carrington MJ, and Trinder J. 2008. Blood pressure and heart rate during continuous experimental sleep fragmentation in healthy adults. Sleep 31:1701-1712.

Dean E, Bloom A, Cirillo M, Hong Q, Jawl B, Jukes J, Nijjar M, Sadovich S, and Bruno SS. 2012. Association between habitual sleep duration and blood pressure and clinical implications: a systematic review. Blood Pressure 21:45-57. 10.3109/08037051.2011.596320

DiBona GF. 2002. Sympathetic nervous system and the kidney in hypertension. Curr Opin Nephrol Hypertens 11:197-200.

Guo J, Fei Y, Li J, Zhang L, Luo Q, and Chen G. 2016. Gender- and age-specific associations between sleep duration and prevalent hypertension in middle-aged and elderly Chinese: a cross-sectional study from CHARLS 
2011-2012. BMJ Open 6:e011770. 10.1136/bmjopen-2016-011770

Haas DC, Foster GL, Nieto FJ, Redline S, Resnick HE, Robbins JA, Young T, and Pickering TG. 2005. Age-dependent associations between sleep-disordered breathing and hypertension: importance of discriminating between systolic/diastolic hypertension and isolated systolic hypertension in the Sleep Heart Health Study. Circulation 111:614-621. 10.1161/01.cir.0000154540.62381.cf

Hashimoto J, Chonan K, Aoki Y, Ugajin T, Yamaguchi J, Nishimura T, Kikuya M, Michimata M, Matsubara M, Araki T, Hozawa A, Ohkubo T, and Imai Y. 2003. Therapeutic effects of evening administration of guanabenz and clonidine on morning hypertension: evaluation using home-based blood pressure measurements. Journal of Hypertension 21:805-811. 10.1097/01.hjh.0000052481.18130.69

Hoshide S, Yano Y, Haimoto H, Yamagiwa K, Uchiba K, Nagasaka S, Matsui Y, Nakamura A, Fukutomi M, Eguchi K, Ishikawa J, and Kario K. 2016. Morning and Evening Home Blood Pressure and Risks of Incident Stroke and Coronary Artery Disease in the Japanese General Practice Population: The Japan Morning Surge-Home Blood Pressure Study. Hypertension 68:54-61. 10.1161/hypertensionaha.116.07201

Hu SS, Kong LZ, Gao RL, Zhu ML, Wang W, Wang YJ, Wu ZS, Chen WW, and Liu MB. 2012. Outline of the report on cardiovascular disease in China, 2010. Biomedical and Environmental Sciences 25:251-256. 10.3967/08953988.2012.03.001

Johnson AW, Hissen SL, Macefield VG, Brown R, and Taylor CE. 2016. Magnitude of Morning Surge in Blood Pressure Is Associated with Sympathetic but Not Cardiac Baroreflex Sensitivity. Frontiers in Neuroscience 10:412. 10.3389/fnins.2016.00412

Joyner MJ, Charkoudian N, and Wallin BG. 2010. Sympathetic nervous system and blood pressure in humans: individualized patterns of regulation and their implications. Hypertension 56:10-16. 10.1161/hypertensionaha.109.140186

Kario K, Pickering TG, Hoshide S, Eguchi K, Ishikawa J, Morinari M, Hoshide Y, and Shimada K. 2004. Morning blood pressure surge and hypertensive cerebrovascular disease: role of the alpha adrenergic sympathetic nervous system. American Journal of Hypertension 17:668-675. 10.1016/j.amjhyper.2004.04.001

Kearney PM, Whelton M, Reynolds K, Muntner P, Whelton PK, and He J. 2005. Global burden of hypertension: analysis of worldwide data. Lancet 365:217-223. 10.1016/s0140-6736(05)17741-1

Li G, Wang H, Wang K, Wang W, Dong F, Qian Y, Gong H, Xu G, Li Y, Pan L, Wang B, Zhu G, and Shan G. 2016 . Prevalence, awareness, treatment, control and risk factors related to hypertension among urban adults in Inner Mongolia 2014: differences between Mongolian and Han populations. BMC Public Health 16:294. 10.1186/s12889-016-2965-5

Li H, Kong F, X J J, Zhang M, Wang A, and Zhang Y. 2016b. Hypertension subtypes and risk of cardiovascular diseases in a Mongolian population, inner Mongolia, China. Clinical and Experimental Hypertension 38:3944. 10.3109/10641963.2015.1060981

Li H, Xu T, Tong W, Liu Y, Zhao L, and Zhang Y. 2008. Comparison of cardiovascular risk factors between prehypertension and hypertension in a Mongolian population, Inner Mongolia, China. Circulation Journal 72:1666-1673.

Lombardi F, and Parati G. 2000. An update on: cardiovascular and respiratory changes during sleep in normal and hypertensive subjects. Cardiovasc Res 45:200-211.

Lusardi P, Zoppi A, Preti P, Pesce RM, Piazza E, and Fogari R. 1999. Effects of insufficient sleep on blood pressure in hypertensive patients: a 24-h study. American Journal of Hypertension 12:63-68. 
357

358

359

360

361

362

363

364

365

366

367

368

369

370

371

372

373

374

375

376

377

378

379

380

381

382

383

384

385

386

387

388

389

390

391

392

Lyhne JM, Laugesen E, Hoyem P, Cichosz S, Christiansen JS, Knudsen ST, Hansen KW, Hansen TK, and Poulsen PL. 2015. Morning blood pressure surge and target organ damage in newly diagnosed type 2 diabetic patients: a cross sectional study. BMC Endocrine Disorders 15:77. 10.1186/s12902-015-0068-4

Meisinger C, Heier M, Lowel H, Schneider A, and Doring A. 2007. Sleep duration and sleep complaints and risk of myocardial infarction in middle-aged men and women from the general population: the MONICA/KORA Augsburg cohort study. Sleep 30:1121-1127.

Mills PJ, Dimsdale JE, Ziegler MG, and Nelesen RA. 1995. Racial differences in epinephrine and beta 2-adrenergic receptors. Hypertension 25:88-91.

Okada Y, Galbreath MM, Shibata S, Jarvis SS, Bivens TB, Vongpatanasin W, Levine BD, and Fu Q. 2013. Morning blood pressure surge is associated with arterial stiffness and sympathetic baroreflex sensitivity in hypertensive seniors. Am J Physiol Heart Circ Physiol 305:H793-802. 10.1152/ajpheart.00254.2013

Rutledge DR. 1991. Are there beta-adrenergic receptor response differences between racial groups? DICP 25:824834.

Sayk F, Becker C, Teckentrup C, Fehm HL, Struck J, Wellhoener JP, and Dodt C. 2007. To dip or not to dip: on the physiology of blood pressure decrease during nocturnal sleep in healthy humans. Hypertension 49:10701076. 10.1161/hypertensionaha.106.084343

Sheppard JP, Hodgkinson J, Riley R, Martin U, Bayliss S, and McManus RJ. 2015. Prognostic significance of the morning blood pressure surge in clinical practice: a systematic review. American Journal of Hypertension 28:30-41. 10.1093/ajh/hpu104

Spiegel K, Leproult R, and Van Cauter E. 1999. Impact of sleep debt on metabolic and endocrine function. Lancet 354:1435-1439. 10.1016/s0140-6736(99)01376-8

Vaidya A, and Forman JP. 2010. Vitamin D and hypertension: current evidence and future directions. Hypertension 56:774-779. 10.1161/hypertensionaha.109.140160

Walters AS, and Rye DB. 2009. Review of the relationship of restless legs syndrome and periodic limb movements in sleep to hypertension, heart disease, and stroke. Sleep 32:589-597.

Wing YK, Zhang J, Ho CK, Au CT, and Li AM. 2010. Periodic limb movement during sleep is associated with nocturnal hypertension in children. Sleep 33:759-765.

Yang CC, Lai CW, Lai HY, and Kuo TB. 2002. Relationship between electroencephalogram slow-wave magnitude and heart rate variability during sleep in humans. Neuroscience Letters 329:213-216.

Zhang HW, Zhang YH, Lu MJ, Tong WJ, and Cao GW. 2007. Comparison of hypertension, dyslipidaemia and hyperglycaemia between buckwheat seed-consuming and non-consuming Mongolian-Chinese populations in Inner Mongolia, China. Clinical and Experimental Pharmacology and Physiology 34:838-844. 10.1111/j.1440-1681.2007.04614.x

Zou D, Grote L, Radlinski J, Eder DN, Lindblad U, and Hedner J. 2009. Nocturnal pulse wave attenuation is associated with office blood pressure in a population based cohort. Sleep Medicine 10:836-843. 10.1016/j.sleep.2008.10.001 


\section{Table $\mathbf{1}$ (on next page)}

Table 1. Demographic and clinical characteristics of study subjects

For continuous variables, data were mean \pm standard deviation(SD); For categorical variables, data were relative frequencies (percentages). BMI= body mass index; $F G=$ fasting blood glucose; $\mathrm{CHO}=$ total cholesterol; $\mathrm{HDL}-\mathrm{C}=$ high density lipoprotein; $\mathrm{LDL}-\mathrm{C}=$ low density lipoprotein; TG=triglyceride; PSQI-I= mild sleep disorder; PSQI-II=moderate sleep disorder; PSQI-III=severe sleep disorder; $\mathrm{DSBP}=$ daytime systolic blood pressure; $\mathrm{DDBP}=$ daytime diastolic blood pressure; NSBP=nocturnal systolic blood pressure; NDBP=nocturnal diastolic blood pressure; $\mathrm{MSBP}=$ morning surge systolic blood pressure; $\mathrm{MDBP}=$ morning surge diastolic blood pressure; D-NE: daytime norepinephrine; D-E: daytime epinephrine; D-DA: daytime dopamine; N-NE: nocturnal norepinephrine; N-E: nocturnal epinephrine; N-DA: nocturnal dopamine; HR: heart rates; D-HR: daytime heart rates; N-HR: nocturnal heart rates. 
Table 1 Demographic and clinical characteristics of study subjects

\begin{tabular}{|c|c|c|c|c|}
\hline & $\begin{array}{l}\text { Mongolian } \\
\text { group }\end{array}$ & $\begin{array}{l}\text { Han group } \\
(\mathrm{n}=115)\end{array}$ & $Z\left(\chi^{2}\right)$ & $\mathrm{P}$ \\
\hline \multicolumn{5}{|c|}{$(n=87)$} \\
\hline \multicolumn{5}{|c|}{ Demographics } \\
\hline Age & $52.01 \pm 10.62$ & $53.23 \pm 9.99$ & -1.105 & 0.269 \\
\hline \multicolumn{5}{|l|}{ Sex } \\
\hline Male & $40(34.78 \%)$ & $34(39.08 \%)$ & 0.394 & 0.530 \\
\hline female & $47(65.12 \%)$ & $81(60.92 \%)$ & & \\
\hline \multicolumn{5}{|l|}{$\left(\mathrm{kg} / \mathrm{m}^{2}\right)$} \\
\hline \multicolumn{5}{|l|}{$(\%)$} \\
\hline \multicolumn{5}{|c|}{ Biochemical Indexes $(\mathrm{mmol} / \mathrm{L})$} \\
\hline FG & $5.18 \pm 0.64$ & $4.99 \pm 0.85$ & -2.328 & 0.019 \\
\hline $\mathrm{CHO}$ & $4.82 \pm 0.93$ & $4.68 \pm 1.06$ & -1.358 & 0.174 \\
\hline TG & $2.18 \pm 0.89$ & $1.69 \pm 0.70$ & -4.002 & 0.273 \\
\hline HDL-C & $1.49 \pm 0.62$ & $1.43 \pm 0.55$ & -0.672 & 0.501 \\
\hline LDL-C & $2.36 \pm 0.97$ & $2.56 \pm 1.02$ & -1.097 & 0.651 \\
\hline \multicolumn{5}{|c|}{ PSQI(\%) } \\
\hline PSQI- I & $42(48.28)$ & $57(49.46)$ & 0.033 & 0.856 \\
\hline PSQI- II & $26(29.89)$ & $29(25.22)$ & 0.545 & 0.461 \\
\hline PSQI-III & $19(21.83)$ & $29(25.22)$ & 0.312 & 0.576 \\
\hline \multicolumn{5}{|c|}{$\mathrm{BP}(\mathrm{mmHg})$} \\
\hline DSBP & $136 \pm 4$ & $131 \pm 8$ & -5.592 & 0.000 \\
\hline DDBP & $86 \pm 8$ & $89 \pm 9$ & -2.761 & 0.006 \\
\hline NSBP & $127 \pm 7$ & $119 \pm 12$ & -5.204 & 0.000 \\
\hline NDBP & $82 \pm 8$ & $80 \pm 10$ & -2.588 & 0.000 \\
\hline MSBP & $163 \pm 7$ & $155 \pm 12$ & -5.542 & 0.000 \\
\hline MDBP & $101 \pm 6$ & $102 \pm 8$ & -1.353 & 0.176 \\
\hline \multicolumn{5}{|c|}{ HR(beats/min) } \\
\hline D-HR & $75 \pm 7$ & $73 \pm 5$ & -0.254 & 0.024 \\
\hline N-HR & $62 \pm 6$ & $65 \pm 5$ & -3.031 & 0.002 \\
\hline \multicolumn{5}{|c|}{ Catecholamine $\left(\mu \mathrm{mol} \cdot \mathrm{L}^{-1}\right)$} \\
\hline D-NE & $0.32 \pm 0.12$ & $0.31 \pm 0.16$ & -1.336 & 0.181 \\
\hline
\end{tabular}




\begin{tabular}{ccccc}
\hline D-E & $0.22 \pm 0.07$ & $0.21 \pm 0.09$ & -0.768 & 0.443 \\
D-DA & $1.14 \pm 0.35$ & $1.01 \pm 0.42$ & -2.726 & 0.006 \\
N-NE & $0.23 \pm 0.09$ & $0.22 \pm 0.11$ & -1.490 & 0.136 \\
N-E & $0.18 \pm 0.06$ & $0.18 \pm 0.07$ & -0.140 & 0.889 \\
N-DA & $0.98 \pm 0.22$ & $1.07 \pm 0.25$ & -2.499 & 0.012 \\
\hline
\end{tabular}

For continuous variables, data were mean \pm standard deviation(SD); For categorical variables, data were relative frequencies (percentages). $\mathrm{BMI}=$ body mass index; $\mathrm{FG}=$ fasting blood glucose; $\mathrm{CHO}=$ total cholesterol; HDL-C=high density lipoprotein; LDL-C=low density lipoprotein; TG=triglyceride; PSQI$\mathrm{I}=$ mild sleep disorder; PSQI- II =moderate sleep disorder; PSQI-III=severe sleep disorder; $\mathrm{DSBP}=$ daytime systolic blood pressure; $\mathrm{DDBP}=$ daytime diastolic blood pressure; $\mathrm{NSBP}=$ nocturnal systolic blood pressure; NDBP=nocturnal diastolic blood pressure; $\mathrm{MSBP}=$ morning surge systolic blood pressure; $\mathrm{MDBP}=$ morning surge diastolic blood pressure; $\mathrm{D}-\mathrm{NE}$ : daytime norepinephrine; $\mathrm{D}-\mathrm{E}$ : daytime epinephrine; D-DA: daytime dopamine ; N-NE: nocturnal norepinephrine; N-E: nocturnal epinephrine; N-DA: nocturnal dopamine; HR: heart rates; D-HR: daytime heart rates; N-HR: nocturnal heart rates. 


\section{Table 2 (on next page)}

Table 2. Demographic characteristics of Mongolian and Han patients among PSQI groups

For categorical variables, data were number of the participates and brackets are percentages. PSQI-I= mild sleep disorder; PSQI-II=moderate sleep disorder; PSQI-III=severe sleep disorder; $1 \mathrm{~h} / \mathrm{d}=1$ hour per day. 
Table 2 Demographic characteristics of Mongolian and Han patients among PSQI groups

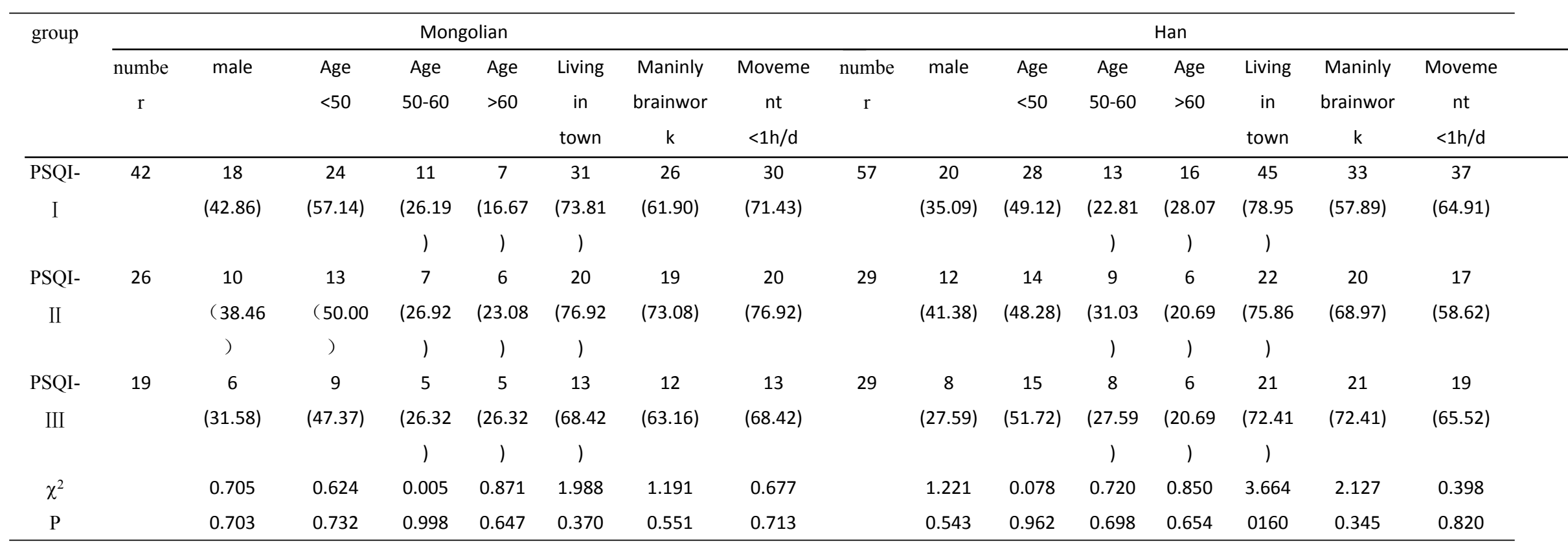

4 For categorical variables, data were number of the participates and brackets are percentages. PSQI- I = mild sleep disorder; PSQI- II =moderate sleep disorder; PSQI-

$5 \mathrm{III}=$ severe sleep disorder; $1 \mathrm{~h} / \mathrm{d}=1$ hour per day 


\section{Table 3(on next page)}

Table 3. PSQI scores between Mongolian and Han groups

For continuous variables, data were mean \pm standard deviation(SD). 
Table 3 PSQI scores between Mongolian and Han groups

2

\begin{tabular}{|c|c|c|c|c|}
\hline & $\begin{array}{l}\text { Mongolian } \\
\text { group } \\
(\mathrm{n}=87)\end{array}$ & $\begin{array}{l}\text { Han group } \\
(\mathrm{n}=115)\end{array}$ & Z & $\mathrm{P}$ \\
\hline Sleep Quality & $2.08 \pm 0.88$ & $2.03 \pm 0.86$ & -0.484 & 0.629 \\
\hline Sleep Time & $2.18 \pm 0.88$ & $2.23 \pm 0.88$ & -0.515 & 0.606 \\
\hline $\begin{array}{c}\text { Sleep } \\
\text { Latency }\end{array}$ & $2.18 \pm 0.87$ & $2.23 \pm 0.88$ & -0.515 & 0.606 \\
\hline $\begin{array}{l}\text { Sleep } \\
\text { Efficiency }\end{array}$ & $2.16 \pm 0.76$ & $2.09 \pm 0.77$ & -0.731 & 0.465 \\
\hline $\begin{array}{c}\text { Sleep } \\
\text { Disturbance }\end{array}$ & $1.14 \pm 0.98$ & $1.06 \pm 0.97$ & -0.558 & 0.577 \\
\hline $\begin{array}{c}\text { Sodium } \\
\text { Amytal Use }\end{array}$ & $1.00 \pm 1.02$ & $0.94 \pm 1.04$ & -0.507 & 0.612 \\
\hline $\begin{array}{c}\text { Daytime } \\
\text { Disfunction }\end{array}$ & $1.82 \pm 0.74$ & $2.10 \pm 0.77$ & -2.565 & 0.010 \\
\hline PSQI Scores & $12.67 \pm 4.40$ & $12.81 \pm 4.42$ & -0.262 & 0.793 \\
\hline
\end{tabular}

3 continuous variables, data were mean \pm standard deviation(SD).

4 


\section{Table 4 (on next page)}

Table 4. The comparison of ABPM and urinary catecholamine in Han nationality among different PSQI groups

For continuous variables, data were mean \pm standard deviation(SD). PSQI-I= mild sleep disorder; PSQI-II=moderate sleep disorder; PSQI-III=severe sleep disorder. DSBP=daytime systolic blood pressure; DDBP=daytime diastolic blood pressure; NSBP=nocturnal systolic blood pressure; NDBP=nocturnal diastolic blood pressure; $\mathrm{MSBP}=$ morning surge systolic blood pressure; MDBP=morning surge diastolic blood pressure. D-NE: daytime norepinephrine; D-E: daytime epinephrine; D-DA: daytime dopamine ; N-NE: nocturnal norepinephrine; N-E: nocturnal epinephrine; N-DA: nocturnal dopamine. 
Table 4 The comparison of ABPM and urinary catecholamine in Han nationality among different PSQI groups

\begin{tabular}{|c|c|c|c|c|c|c|c|c|c|c|c|c|c|}
\hline group & number & $\begin{array}{c}\text { DSBP } \\
(\mathrm{mmHg}) \\
\end{array}$ & $\begin{array}{c}\text { DDBP } \\
(\mathrm{mmHg})\end{array}$ & $\begin{array}{c}\text { NSBP } \\
(\mathrm{mmHg})\end{array}$ & $\begin{array}{c}\text { NDBP } \\
(\mathrm{mmHg})\end{array}$ & $\begin{array}{c}\text { MSBP } \\
(\mathrm{mmHg})\end{array}$ & $\begin{array}{c}\mathrm{MDBP} \\
(\mathrm{mmHg})\end{array}$ & $\begin{array}{c}\mathrm{D}-\mathrm{NE} \\
\mu \mathrm{mol} \cdot \mathrm{L}^{-1}\end{array}$ & $\begin{array}{c}\text { D-E } \\
\mu \mathrm{mol} \cdot \mathrm{L}^{-1}\end{array}$ & $\begin{array}{c}\text { D-DA } \\
\mu \mathrm{mol} \cdot \mathrm{L}^{-1}\end{array}$ & $\begin{array}{c}\mathrm{N}-\mathrm{NE} \\
\mu \mathrm{mol} \cdot \mathrm{L}^{-1}\end{array}$ & $\begin{array}{c}\mathrm{N}-\mathrm{E} \\
\mu \mathrm{mol} \cdot \mathrm{L}^{-1}\end{array}$ & $\begin{array}{c}\mathrm{N}-\mathrm{DA} \\
\mu \mathrm{mol} \cdot \mathrm{L}^{-1}\end{array}$ \\
\hline $\begin{array}{c}\text { PSQI- } \\
\text { I }\end{array}$ & 57 & $128 \pm 9$ & $87 \pm 9$ & $112 \pm 11$ & $78 \pm 6$ & $150 \pm 11$ & $101 \pm 7$ & $0.20 \pm 0.08$ & $0.18 \pm 0.08$ & $0.75 \pm 0.23$ & $0.15 \pm 0.05$ & $0.17 \pm 0.07$ & $0.96 \pm 0.24$ \\
\hline $\begin{array}{c}\text { PSQI- } \\
\text { II }\end{array}$ & 29 & $132 \pm 5$ & $90 \pm 6$ & $122 \pm 8$ & $80 \pm 15$ & $154 \pm 7$ & $104 \pm 7$ & $0.35 \pm 0.09$ & $0.23 \pm 0.07$ & $1.05 \pm 0.20$ & $0.27 \pm 0.11$ & $0.18 \pm 0.06$ & $1.08 \pm 0.19$ \\
\hline $\begin{array}{c}\text { PSQI- } \\
\text { III }\end{array}$ & 29 & $136 \pm 4$ & $91 \pm 7$ & $130 \pm 5$ & $83 \pm 9$ & $167 \pm 8$ & $104 \pm 9$ & $0.48 \pm 0.17$ & $0.27 \pm 0.10$ & $1.50 \pm 0.41$ & $0.29 \pm 0.12$ & $0.21 \pm 0.07$ & $1.27 \pm 0.21$ \\
\hline$\chi^{2}$ & & 31.942 & 4.491 & 53.453 & 11.796 & 46462 & 4.482 & 61.855 & 17.662 & 58.111 & 36.346 & 4.788 & 26.628 \\
\hline $\mathrm{P}$ & & 0.000 & 0.106 & 0.000 & 0.003 & 0.000 & 0.106 & 0.000 & 0.000 & 0.000 & 0.000 & 0.091 & 0.000 \\
\hline
\end{tabular}

5 For continuous variables, data were mean \pm standard deviation(SD). PSQI- I = mild sleep disorder; PSQI- II =moderate sleep disorder; PSQI-III=severe

6 sleep disorder. $\mathrm{DSBP}=$ daytime systolic blood pressure; $\mathrm{DDBP}=$ daytime diastolic blood pressure; NSBP=nocturnal systolic blood pressure;

$7 \mathrm{NDBP}=$ nocturnal diastolic blood pressure; $\mathrm{MSBP}=$ morning surge systolic blood pressure; $\mathrm{MDBP}=$ morning surge diastolic blood pressure. $\mathrm{D}-\mathrm{NE}$ :

8 daytime norepinephrine; D-E: daytime epinephrine; D-DA: daytime dopamine ; N-NE: nocturnal norepinephrine; N-E: nocturnal epinephrine; N-DA:

9 nocturnal dopamine. 


\section{Table 5 (on next page)}

Table 5. The comparison of ABPM and urinary catecholamine in Mongolian among different PSQI groups

For continuous variables, data were mean \pm standard deviation(SD). PSQI-I= mild sleep disorder; PSQI-II=moderate sleep disorder; PSQI-II=severe sleep disorder. DSBP=daytime systolic blood pressure; DDBP=daytime diastolic blood pressure; NSBP=nocturnal systolic blood pressure; NDBP=nocturnal diastolic blood pressure; $\mathrm{MSBP}=$ morning surge systolic blood pressure; MDBP=morning surge diastolic blood pressure. D-NE: daytime norepinephrine; D-E: daytime epinephrine; D-DA: daytime dopamine ; N-NE: nocturnal norepinephrine; N-E: nocturnal epinephrine; N-DA: nocturnal dopamine. 
Table 5 The comparison of ABPM and urinary catecholamine in Mongolian among different PSQI groups

\begin{tabular}{|c|c|c|c|c|c|c|c|c|c|c|c|c|c|}
\hline group & number & $\begin{array}{c}\text { DSBP } \\
(\mathrm{mmHg}) \\
\end{array}$ & $\begin{array}{c}\text { DDBP } \\
(\mathrm{mmHg})\end{array}$ & $\begin{array}{c}\text { NSBP } \\
(\mathrm{mmHg}) \\
\end{array}$ & $\begin{array}{c}\text { NDBP } \\
(\mathrm{mmHg}) \\
\end{array}$ & $\begin{array}{c}\text { MSBP } \\
(\mathrm{mmHg})\end{array}$ & $\begin{array}{c}\mathrm{MDBP} \\
(\mathrm{mmHg})\end{array}$ & $\begin{array}{c}\text { D-NE } \\
\mu \mathrm{mol} \cdot \mathrm{L}^{-1}\end{array}$ & $\begin{array}{c}\text { D-E } \\
\mu \mathrm{mol} \cdot \mathrm{L}^{-1}\end{array}$ & $\begin{array}{c}\text { D-DA } \\
\mu \mathrm{mol} \cdot \mathrm{L}^{-1}\end{array}$ & $\begin{array}{c}\text { N-NE } \\
\mu \mathrm{mol} \cdot \mathrm{L}^{-1}\end{array}$ & $\begin{array}{c}\mathrm{N}-\mathrm{E} \\
\mu \mathrm{mol} \cdot \mathrm{L}^{-1}\end{array}$ & $\begin{array}{c}\mathrm{N}-\mathrm{DA} \\
\mu \mathrm{mol} \cdot \mathrm{L}^{-1}\end{array}$ \\
\hline $\begin{array}{c}\text { PSQI- } \\
\text { I }\end{array}$ & 42 & $135 \pm 3$ & $85 \pm 7$ & $125 \pm 5$ & $81 \pm 9$ & $162 \pm 8$ & $100 \pm 7$ & $0.27 \pm 0.09$ & $0.20 \pm 0.05$ & $1.03 \pm 0.33$ & $0.22 \pm 0.08$ & $0.17 \pm 0.06$ & $0.99 \pm 0.25$ \\
\hline $\begin{array}{c}\text { PSQI- } \\
\text { II }\end{array}$ & 26 & $136 \pm 3$ & $86 \pm 9$ & $127 \pm 8$ & $82 \pm 7$ & $163 \pm 7$ & $101 \pm 5$ & $0.37 \pm 0.11$ & $0.24 \pm 0.08$ & $1.07 \pm 0.17$ & $0.22 \pm 0.09$ & $0.19 \pm 0.06$ & $0.93 \pm 0.21$ \\
\hline $\begin{array}{c}\text { PSQI- } \\
\text { III }\end{array}$ & 19 & $136 \pm 4$ & $88 \pm 8$ & $131 \pm 6$ & $83 \pm 7$ & $168 \pm 6$ & $102 \pm 7$ & $0.35 \pm 0.14$ & $0.24 \pm 0.08$ & $1.48 \pm 0.40$ & $0.25 \pm 0.12$ & $0.18 \pm 0.06$ & $1.00 \pm 0.17$ \\
\hline$\chi^{2}$ & & 0.170 & 1.914 & 14.471 & 0.231 & 7.622 & 0.232 & 13.606 & 9.022 & 17.715 & 1.214 & 1.686 & 0.765 \\
\hline $\mathrm{P}$ & & 0.918 & 0.384 & 0.001 & 0.891 & 0.022 & 0.890 & 0.001 & 0.011 & 0.000 & 0.545 & 0.431 & 0.682 \\
\hline
\end{tabular}

5 For continuous variables, data were mean \pm standard deviation(SD). PSQI- I = mild sleep disorder; PSQI- II =moderate sleep disorder; PSQI-III=severe

6 sleep disorder. $\mathrm{DSBP}=$ daytime systolic blood pressure; $\mathrm{DDBP}=$ daytime diastolic blood pressure; $\mathrm{NSBP}=$ nocturnal systolic blood pressure;

$7 \mathrm{NDBP}=$ nocturnal diastolic blood pressure; $\mathrm{MSBP}=$ morning surge systolic blood pressure; $\mathrm{MDBP}=$ morning surge diastolic blood pressure. $\mathrm{D}-\mathrm{NE}$ :

8 daytime norepinephrine; D-E: daytime epinephrine; D-DA: daytime dopamine ; N-NE: nocturnal norepinephrine; N-E: nocturnal epinephrine; N-DA:

9 nocturnal dopamine. 


\section{Table 6(on next page)}

Table 6. The comparison of ABPM and urinary catecholamine between Mongolian and Han nationality in different PSQI groups

For continuous variables, data were mean \pm standard deviation(SD). PSQI-I= mild sleep disorder; PSQI-II=moderate sleep disorder; PSQI-III=severe sleep disorder. DSBP=daytime systolic blood pressure; DDBP=daytime diastolic blood pressure; NSBP=nocturnal systolic blood pressure; NDBP=nocturnal diastolic blood pressure; $\mathrm{MSBP}=$ morning surge systolic blood pressure; MDBP=morning surge diastolic blood pressure. D-NE: daytime norepinephrine; D-E: daytime epinephrine; D-DA: daytime dopamine ; N-NE: nocturnal norepinephrine; N-E: nocturnal epinephrine; N-DA: nocturnal dopamine. 
Table 6 The comparison of ABPM and urinary catecholamine between Mongolian and Han nationality in different PSQI groups

4

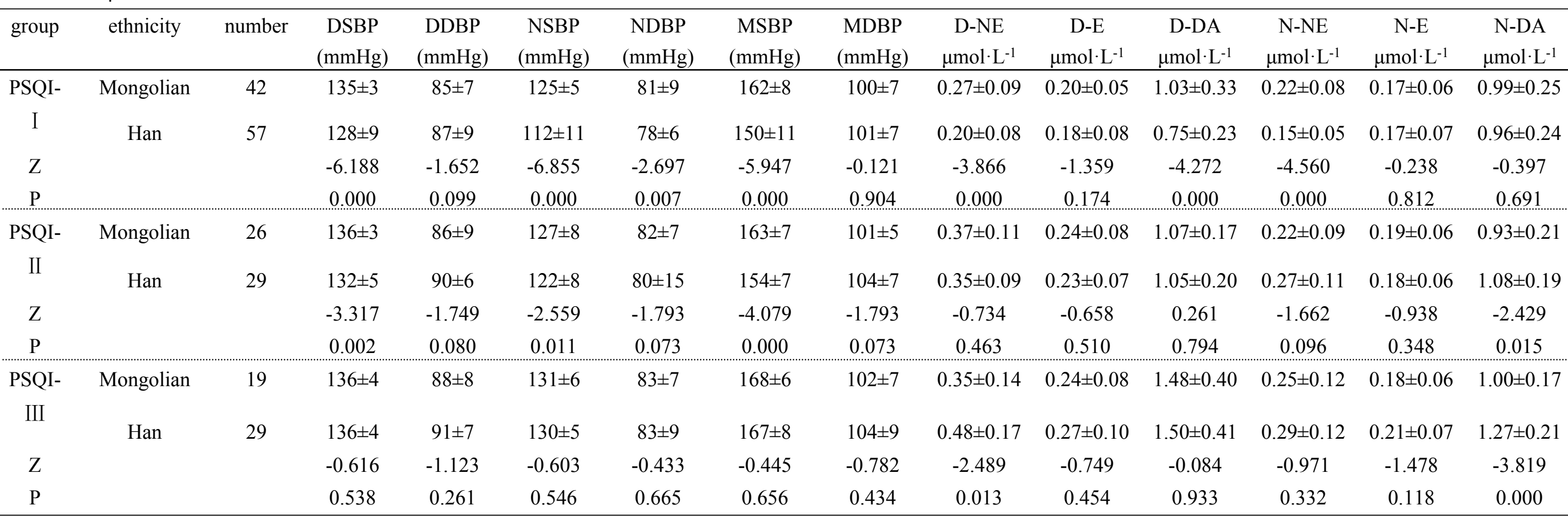

5 For continuous variables, data were mean \pm standard deviation(SD). PSQI- I = mild sleep disorder; PSQI- II =moderate sleep disorder; PSQI-III=severe

6 sleep disorder. $\mathrm{DSBP}=$ daytime systolic blood pressure; $\mathrm{DDBP}=$ daytime diastolic blood pressure; NSBP=nocturnal systolic blood pressure;

$7 \mathrm{NDBP}=$ nocturnal diastolic blood pressure; $\mathrm{MSBP}=$ morning surge systolic blood pressure; $\mathrm{MDBP}=$ morning surge diastolic blood pressure. $\mathrm{D}-\mathrm{NE}$ :

8 daytime norepinephrine; D-E: daytime epinephrine; D-DA: daytime dopamine ; N-NE: nocturnal norepinephrine; N-E: nocturnal epinephrine; N-DA:

9 nocturnal dopamine. 


\section{Figure 1 (on next page)}

Figure 1. Study flow chart of subjects

$\mathrm{ABPM}=$ ambulatory blood pressure monitoring. 
692 participatied the study

281 selected for $\mathrm{PSQI} \geqslant 7$

Excluded

25 had incomplete de mographic data

256 included for inspection study

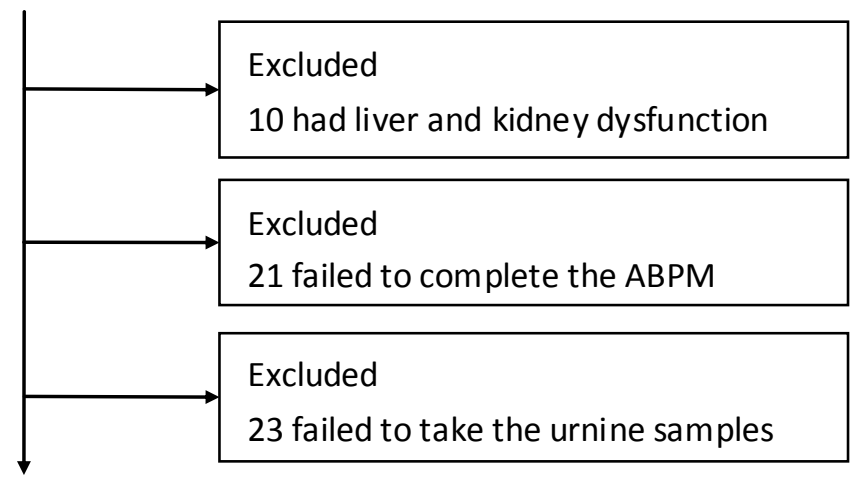

202 included for final study

(87 Mongolian and 115 Han subiets) 


\section{Figure 2}

Figure 2. 24-hour blood pressure and heart rate between Mongolian and Han groups

A: 24-hour systolic blood pressure(SBP) between Mongolian and Han groups; B: 24-hour diastolic blood pressure(DBP) between Mongolian and Han groups; C: 24-hour heart rate(HR) between Mongolian and Han groups. 
$\mathbf{A}$

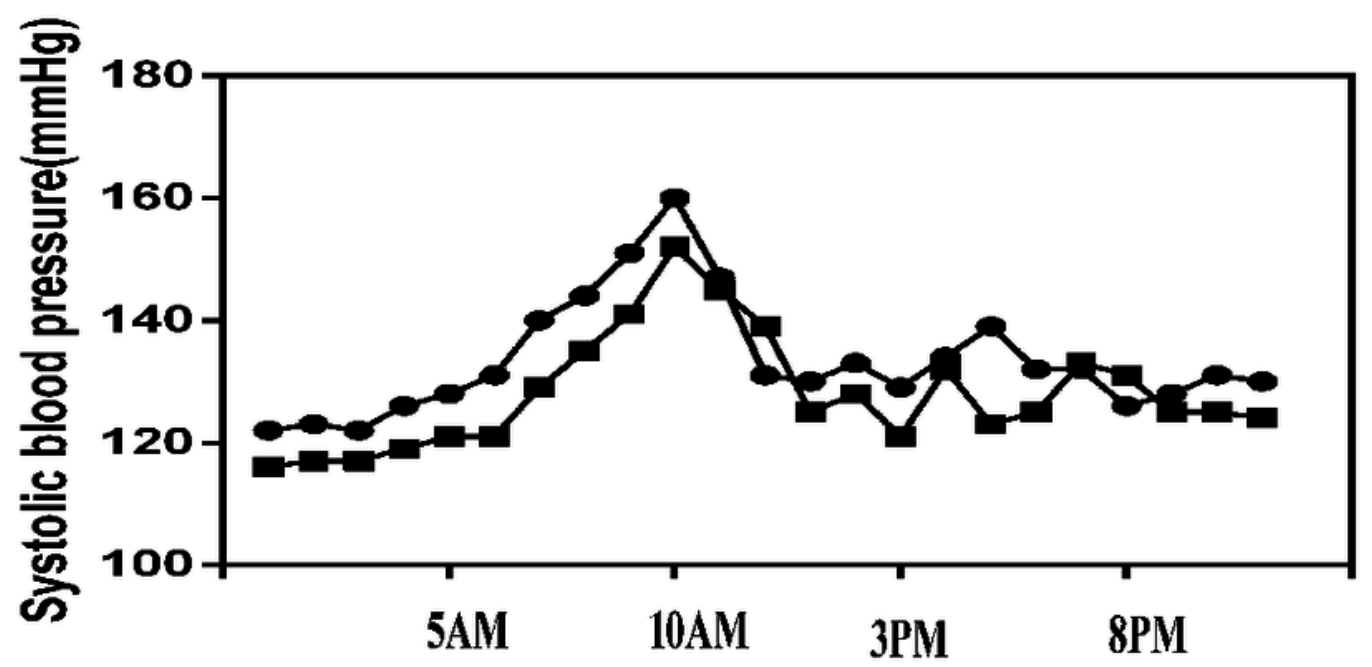

$\mathbf{B}$

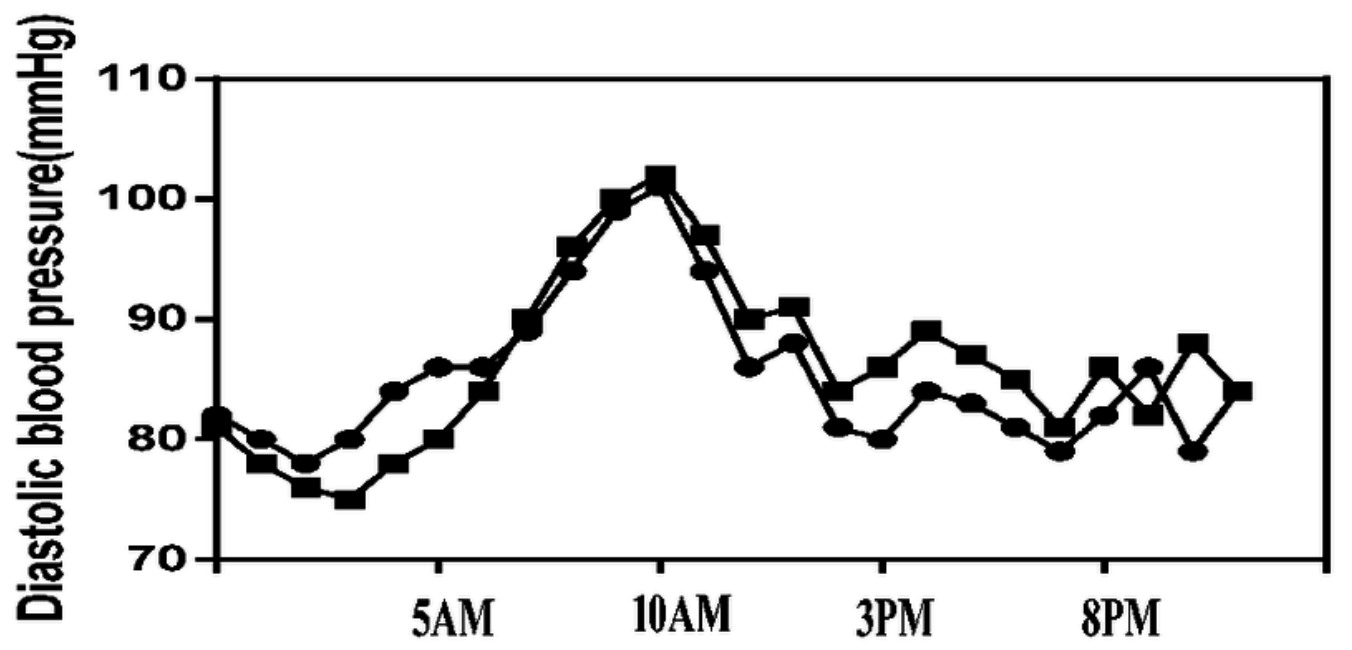

C

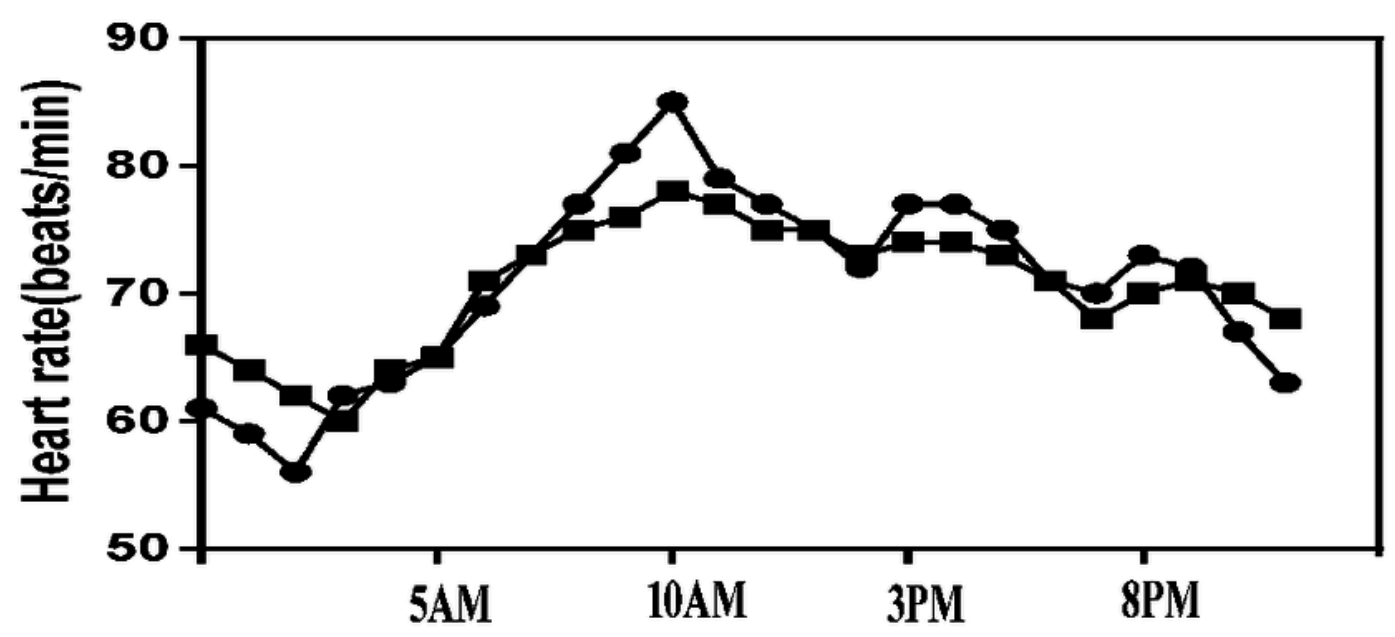




\section{Figure 3}

Figure 3. The correlation between MSBP and catecholamines

A: the correlation between MSBP and D-DA in Mongolian group; $B$ : the correlation between MSBP and D-NE in Han group; C: the correlation between MSBP and D-E in Han group; D: the correlation between MSBP and D-DA in Han group; E: the correlation between MSBP and N-NE in Han group; F: the correlation between MSBP and N-DA in Han group. MSBP=morning surge systolic pressure. MSBP=morning surge systolic blood pressure; D-DA: daytime dopamine; DNE: daytime norepinephrine; D-E: daytime epinephrine; N-NE: nocturnal norepinephrine; NDA: nocturnal dopamine. 

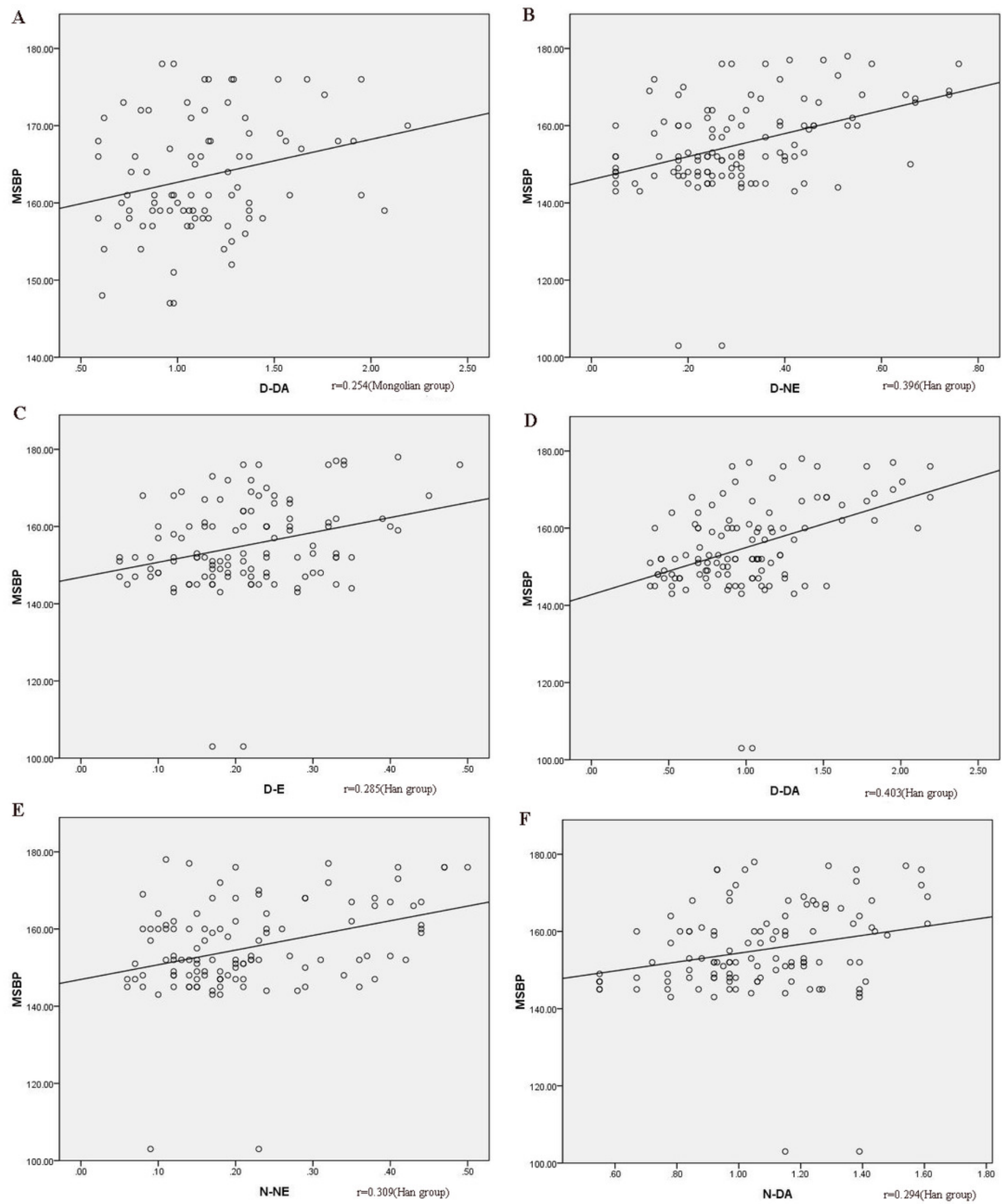